\title{
An Integrated Approach for Segmentation and Representation of Range Images
}

Olga R. P. Bellon

UFPR-Depto. de Informática

81513-970 Curitiba-PR-Brazil

olga@inf.ufpr.br

\author{
Clesio L. Tozzi \\ UNICAMP-FEEC-DCA \\ 13081-970 Campinas-SP-Brazil \\ clesio@dca.fee.unicamp.br
}

\begin{abstract}
This paper presents an integrated approach for segmentation and representation of objects in range images. The segmentation is based on the association of edge detection with clustering techniques, and it produces a set of labeled regions plus the coefficients of the plane fitted to each region. With this information, the reconstruction error for the whole image is estimated, and the initial number of regions supplied to the clustering algorithm can be updated, based on this error. Once the image reconstruction error is smaller than the desired, the next step is to create a polyhedral representation to the surfaces of the image. For each segmented region, the representation process yields its 3D vertices' coordinates, ordered to form a polygon. The final representation is robust enough to guarantee that there are no cracks on the reconstructed surfaces. The main contributions of this work are: (1) A new approach for determining the optimal number of image regions; (2) A suitable representation that can be applied to both polyhedral and nonpolyhedral objects.
\end{abstract}

\section{Introduction}

In image analysis, it is usually convenient to transform the results of the segmentation into a more suitable form, for instance, to perform interpretation and recognition of objects. This representation must be robust enough to allow the object reconstruction with a small error. Since the representation is tightly related to the segmentation results, they should have a combined treatment.

This paper presents an integrated method for segmentation and representation of objects in range images. The segmentation is based on the $K$-Means clustering technique [4] which is combined with edge detection information, yielding a set of segments well approximated by planes. The representation makes use of these segments to build a suitable polyhedral description

The main contributions of this work are: (1) A new approach for determining the optimal number of image regions; (2) A suitable representation that can be applied to both polyhedral and non-polyhedral objects.

This work is organized as follows. Section 2 presents a brief discussion on the related work. In Section 3, the segmentation/representation process is presented. Finally, in Section 4, the experimental results are shown, followed by our conclusions.

\section{Related Works}

Clustering techniques show great independence from rigid threshold values, as compared with other segmentation techniques like region growing [1] and split-andmerge [5],[12]. However, in clustering algorithms, it is necessary to determine the ideal number of image regions. There possibilities to find this number are: (a) Splitting/merging analysis after the clustering process; (b) Generating various sets of clusters, one for each possible number of regions, and to develop a methodology to determine what set best represents the image. Possibility (b) is inefficient in many 
applications. Possibility (a) suffers from the same problem related to other techniques: the threshold determination.

Two relevant research lines [8],[9] use possibility (b). They analyze the segmentation results to determine the optimal number of clusters to the image based on within-cluster and between-clusters measures. However, these two methods are computationally costly, and none of them guarantee the correct determination of the searched number. Bhandarkar and Siebert [2] use a clustering algorithm to segment range images of polyhedral objects. The number of regions is adopted as the maximum expected in the image, and the clustering result is analyzed to identify regions that can be splitted/merged, based on experimental thresholds.

After the segmentation is done, the next step in the image analysis is to create a robust representation to the segmented regions. This representation must allow image reconstruction with small error, as well as be suitable to image interpretation. The representation is tightly related to the segmentation results but such association, from the topological viewpoint, has been poorly contemplated in the literature.

Some researchers deal with image segmentation and representation [1],[8],[12]. However, they do not treat the representation problem in terms of the topology of the segmented regions. Bhandarkar and Siebert [2] create a topological representation of segmented objects in range images, but their work is limited only to polyhedral objects. Faugueras and Herbert [5] present an approach to solve the representation problem of segmented images by using a triangulation processes, but the obtained representation is computationally costly.

The segmentation/representation method developed is presented in the next section.

\section{The Method of Segmentation/Representation}

To solve the problem of determining the optimal number of image regions, we propose to start the clustering process by using an initial estimate of this number, and to update it based on the clustering results without rigid threshold values. This estimate is supplied by performing an edge detection process in the range image. We also propose a simple and efficient method to create an approximate, topologically driven, polyhedral representation for both polyhedral objects and non-polyhedral objects in segmented range images. The method is based on the assumption that the object segments are well approximated by planes, in accordance with the segmentation process used. For each segment (region), the method yields the 3D vertices' coordinates, ordered to form a polygon, and the region's fitted plane equation. This representation is easily built from the range information, and it yields a fast image reconstruction, as shown below.

\subsection{The Segmentation Process}

\subsubsection{Edge Detection and Normal Computation}

First of all, the range image undergoes an edge detection process to identify jump and roof edges. In depth edges there is a significant discontinuity of depth values between neighbouring pixels. In roof edges there is a significant variation in the normal orientation of the adjacent surfaces.

To identify the jump edge pixels, a common border operator can be used. We used the DRF (Direct Recursive Filter) operator, developed by Chen and Castan [5]. The 
threshold $T_{1}$ used to define the edge pixels is obtained based on [7]. It is equal to the average $T_{m}$ added by 1.5 plus the standard deviation $\sigma$ of all the resulting values from the DRF application.

The roof edges are detected by a more complex process. In this work, we compute the normal parameters to each pixel and, from this information, identify the roof edges by the variation of the normal orientation angles. The normal $(a, b, c)$ to each pixel $(i, j)$ is computed by a plane fitting process, using the Least-Mean-Square method [10], in a $3 \times 3$ window centered in pixel $p$. In this computation, the jump edge pixels already detected are not taken into account to reduce spurious effects. The angle that measures the normal change between a pixel and its neighbours is given by the greater value of its normal variation related to its neighbours. These values are calculated from the normal vectors' inner product. When this angle is greater than a threshold $T_{2}$, which is set experimentally to 10 degrees, the pixel is considered to belong to a roof edge.

Once the jump edges and the roof edges are detected, the edge map can be created. From the edge map, after it suffers an edge closing process, it is possible to estimate the initial number $K$ of regions by counting the number of its closed regions. This number is then supplied to the clustering algorithm.

\subsubsection{Approximation Error Computation}

The computation of the normals to each pixel is based on a $3 \times 3$ window, and it contains an error related to the process of fitting a plane to the window. This error, named approximation error $\varepsilon_{a}$, can be estimated to each image pixel $p$ by:

$$
\varepsilon_{u}^{2}(p)=\frac{1}{n_{e}} \sum_{i \in Y}\left(d_{p}-a_{p} x_{i}-b_{p} y_{i}-c_{p} z_{i}\right)^{2}
$$

where $a_{p}, b_{p}, c_{p}, d_{p}$ are the plane coefficients at pixel $p,\left(x_{t}, y_{1}, z_{1}\right)$ are the 3D coordinates of the pixels belonging to the neighbourhood $v$ of pixel $p$, and $n_{e}$ is the number of pixels in the neighbourhood $v$ that contribute to its normal computation. The global approximation error is the mean of all approximation errors, and can be computed by:

$$
\varepsilon_{a}=\frac{1}{n-n} \sum \varepsilon_{a}^{2}(p)
$$

where $n_{b}$ is the total number of image edge pixels. The global approximation error will be used as a parameter to estimate the quality of the next phase of the process.

\subsubsection{Clustering}

The basic concepts of the clustering algorithm adopted [6] are described next.

Let the $i$-th pattern, $i=1,2, \ldots, n$, from the data set under observation be written as $x_{i}=\left(x_{i 1}, x_{i 2}, \ldots, x_{i N}\right)^{T}$, where $\left(x_{i 1}, x_{i 2}, \ldots, x_{i N}\right)^{T}$ is its feature vector and $\mathcal{N}$ is the number of features. The number of patterns, $n$ is assumed to be significantly larger than $\mathcal{N}$. A clustering is a partition $\left[C_{1}, C_{2}, \ldots, C_{K}\right]$ of the integers $[1,2, \ldots, n]$ that assigns each pattern a single cluster label. The patterns corresponding to the integers in $C_{k}$ form the $k$-th cluster, whose center is: $c_{k}=\left(c_{k 1}, c_{k 2}, \ldots, c_{k N}\right)^{T}$, where 


$$
c_{k j}=\frac{1}{M_{k}} \sum_{i \in C_{K}} x_{i j}
$$

and $M_{k}$ is the number of patterns in cluster $k$. The squared error for cluster $k$ is:

$$
e_{k}^{2}=\sum_{i \in C_{K}}\left(x_{i}-c_{k}\right)^{T}\left(x_{i}-c_{k}\right)
$$

and the squared error for the clustering is:

$$
E_{K}^{2}=\sum_{k=1}^{K} e_{k}^{2}
$$

The objectives of such a clustering process are to define, for a given $K$, a clustering that minimizes $E_{K}^{2}$ and to find a suitable $K$, much smaller than $n$.

The clustering algorithm applied in the method described herein has the normal vector components as input features. The initial number of regions, $K$, is also provided, based on the edge map. After the clustering algorithm is applied, the obtained result is a segmented image, for which each pixel has a label associated to a connected region. We wish that each segmented region has a small error when approximated by a plane.

\subsubsection{Plane Equations Computation}

The plane fitting process follows the same idea applied to estimate the normals. In this case, all the pixels that belong to each region, without taking into account the edge pixels, are used to compute the plane equation coefficients $\left(a_{k}, b_{k}, c_{k}, d_{k}\right)$ most suitable to each region $K$.

\subsubsection{Reconstruction Error Computation}

The reconstruction error $\varepsilon_{r}$ is computed to each non-edge pixel, based on the region plane equations, by Equation 3.7. Based on this equation, it is possible to compute the global reconstruction error $\varepsilon_{g r}$, and the reconstruction error to each region $K$, given respectively by Equations 3.8 and 3.9, where $n_{b}$ is the total number of image pixels that belong to an edge, and $n_{k}$ is the number of effective pixels, without taking into account the edge pixels, which belong to each region $K$. These equations are similar to those presented by Besl and Jain [1] but equations 3.7-3.9 are adapted to planar approximations.

$$
\begin{aligned}
& \varepsilon_{r}^{2}(p)=\left(d_{k}-a_{k} x_{i}-b_{k} y_{i}-c_{k} z_{i}\right)^{2} \\
& \varepsilon_{g r}^{2}=\frac{1}{n-n_{b}} \sum \varepsilon_{r}^{2}(p) \\
& \varepsilon_{K}^{2}=\frac{1}{n_{k}} \sum_{r \in K}\left(d_{k}-a_{k} x_{i}-b_{k} y_{i}-c_{k} z_{i}\right)^{2}
\end{aligned}
$$

If the global reconstruction error obtained is greater than desired, as based on the approximation error, the number of regions, $K$, can be increased and the clustering process is reapplied. Otherwise, the next step is to build the polyhedral representation. 


\subsection{The Representation Process}

The input information to the representation process is a labeled segmented image. These segments are approximated by planes, and must be grouped to compose one or more objects. To create a robust polyhedral representation from this information, this representation must satisfy the polyhedral surface conditions [11]. The surface must be divided in planar faces that have edges as its intersections. The segments will be approximated by polygons, and their intersections will be line segments. For these conditions to be sufficient, it is necessary to consider the intersections between adjacent segments. The representation process steps are presented below.

\subsubsection{Vertices and Edges Computation}

In the context of this work, a vertex is defined by a pixel $p$ which has three or four different regions on its neighbourhood, including its own region, using 4-neighbourhood and a $3 \times 3$ window centered at the pixel $p$. When a vertex is defined, a set of edges is also defined, with one edge corresponding to each pair of vertices neighbour segments. The first step to build the desired representation is to identify the vertices'neighbour regions. This can be done by using a scan image process, analyzing the neighbouhood of the pixels which have three or four different neighbour regions.

Because of the planar approximation, the 3D coordinates that best represent each vertex may not be exactly those related to the pixel identified. The suitable coordinates must be computed by considering the intersections between neighbour planes in the vertex. Three situations must be considered to compute the best 3D coordinates to each representation vertex: (a) A vertex is formed by the intersection of three-neighbour segments; (b) A vertex is formed by the intersection of fourneighbour segments; and (c) A vertex belongs to a depth edge.

In the case (a), the 3D vertices' coordinates can be computed by solving a simple linear equation system, using the plane equations of the three neighbour segments. Then, the computed vertex must be inserted in the vertices list and the three edges must be inserted in the edges list. When the same idea is used to solve case (b), the linear equation system may be undetermined, because the number of plane equations is greater than the number of variables. Thus, a vertex composed by four segments must be transformed in two vertices linked by an edge, where each vertex is associated to three different segments. In this case, the two vertices must be inserted in the vertices list, after the computation of their 3D coordinates, and their corresponding edges must be inserted in the edges list. To compute the vertices in case (c), the discontinuity between their neighbour segments must be considered. The detected vertex must be inserted in the vertices list without the refinement of its 3D coordinates, because it is not a result of the intersection of their neighbour segments, and the corresponding edges must be inserted in the edges list.

Note that, in accordance to the segmentation process applied to the image, both the depth edges and the roof edges will always keep their original shape. Then, if these edges are not straight lines, they are unsuitable to be directly approximated by our representation of edges, since much information may be lost along the borders. The solution adopted is to perform polygonalization on the edges identified in the edge detection process. This polygonalization produces a set of edges and its two corresponding vertices. After the polygonalization, the new vertices and edges are inserted in the vertices list and in the edges list, respectively. 
The main steps of the algorithm to construct the polyhedral representation are:

1. Perform the polygonalization on the edge map;

2. For each polygon edge do:

2.1. For each edge vertex do:

2.1.1. If the vertex is not inserted in the vertices list do:

2.1.1.1. Insert the vertex in the vertices list;

2.2. Insert the edge in edges list;

3. Scan the segmented image to detect vertex candidates;

3.1. For each detected pixel do:

3.1.I. If the pixel is not inserted in the vertices list:

3.1.1.1. If the pixel has four different neighbour regions:

3.1.1.1.1. Split the pixel in two vertices, as shown in Figure 3.1;

3.1.1.2. For each vertex do:

3.1.1.2.1. If its neighbour regions have any depth discontinuities do:

3.1.1.2.1.1. Compute its new $3 D$ coordinates;

3.1.1.2.2. Insert its $3 D$ coordinates in the vertices list and the edge in the edges list;

4. Evaluate the vertices list:

4.1. If there are two or more vertices too close do:

4.1.1. To merge those vertices;

4.1.2. To reorganize the vertices list and the edges list.

5. Build the regions list, based in the vertices list and in the edges list.

\subsubsection{Representation Quality Measure}

Once an image representation is created, its quality can be measured based on the corresponding initial range image. To compute this measure, the representation obtained must be placed in spatial correspondence with the input range image. This matching can be performed by a calibration process [6], since the correspondence between the 3D points and the image points is known.

After the calibration, the 3D vertices' coordinates of the obtained representation are projected and the corresponding edges are built, to create a 2-D wire-frame image. This image can be used as a visual measure of the representation quality. A quantitative measure of the representation quality can be computed based on the difference between the original 3D vertices' coordinates of the range image and the 3D vertices' coordinates of the obtained representation.

In the next section, we present our experimental results.

\section{Experimental Results}

To illustrate the experimental results, we present a set of images and one Table.

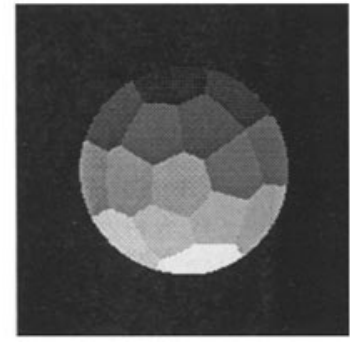

(a) Segmented image $\mathrm{K}=15$.

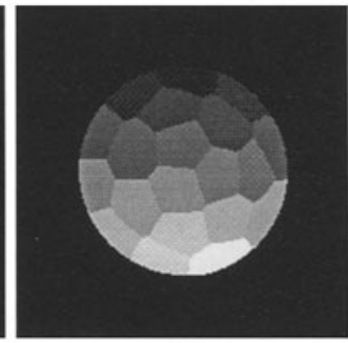

(b) Segmented image $\mathrm{K}=20$.

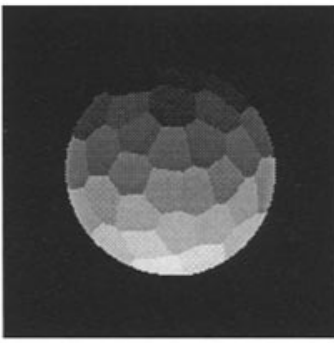

(c) Segmented image $\mathrm{K}=30$.

Figure 4.1: Segmentation results (sphere without noise). 


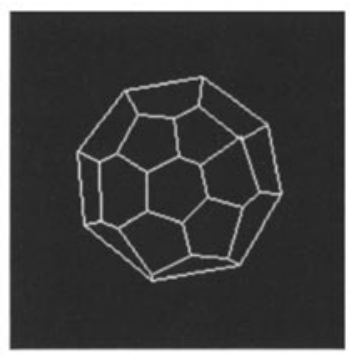

(a) Wire-frame image $\mathrm{K}=15$.

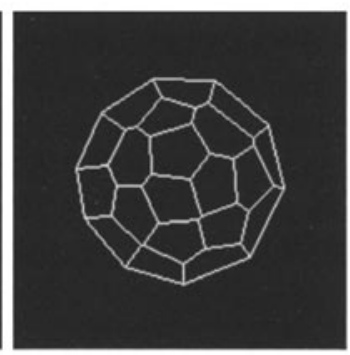

(b) Wire-frame image $K=20$

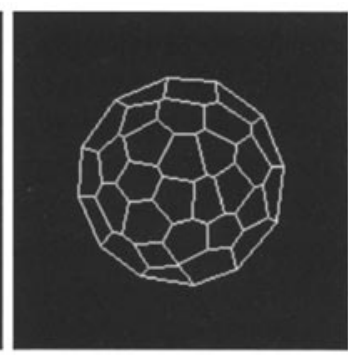

(d) Wire-frame image $\mathrm{K}=30$. Figure 4.2: Wire-frame results (sphere without noise).

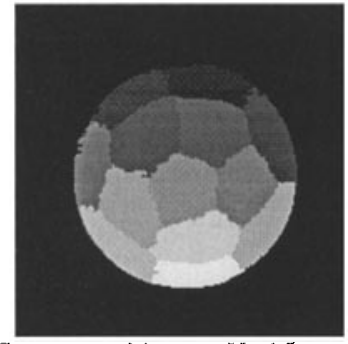

(a) Segmented image $K=15$.

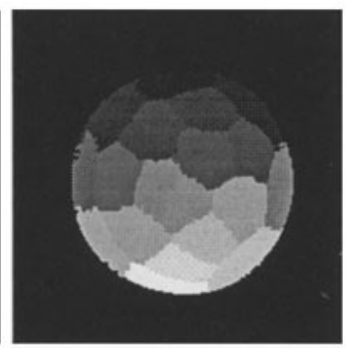

(b) Segmented image $K=20$

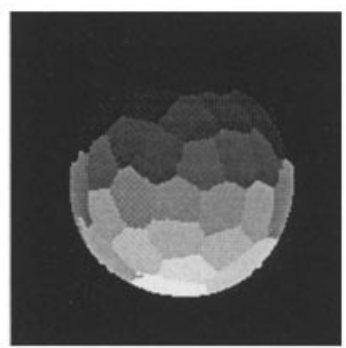

(c) Segmented image $\mathrm{K}=30$ Figure 4.3: Wire-frame results (sphere added by Gaussian noise).

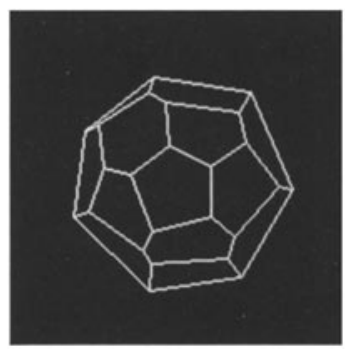

(a) Wire-frame mage $K^{\prime}=15$.

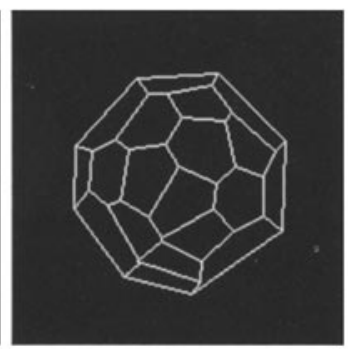

(b) Wire-frame image $K=20$. (c) Wire-frame image $K=30$ ).

Figure 4.4: Wore-frame results (nphere added hy Gaussian noise).

Table 1: Comparison of measure crrors

\begin{tabular}{|c||c|c|c|c|}
\hline Image & $\varepsilon g a$ & $\varepsilon g r$ & $\varepsilon v$ & $\varepsilon v / n v$ \\
\hline \hline Sphere $K=15$ & 0.001574 & 0.455996 & 120.63270 & 4.79 \\
\hline Sphere $K=20$ & 0.001574 & 0.324371 & 97.755966 & 2.57 \\
\hline Sphere $K=30$ & 0.001574 & 0.225245 & 76.374603 & 1.32 \\
\hline Noisy Sphere $K=15$ & 0.006114 & 1.516631 & 400.29523 & 16.68 \\
\hline Noisy Sphere $K=20$ & 0.006114 & 1.208808 & 262.17148 & 7.28 \\
\hline Noisy Sphere $K=30$ & 0.006114 & 0.978072 & 272.94876 & 5.45 \\
\hline
\end{tabular}

Figure 4.1(a)-(c) presents the segmentation results for a range image of a sphere. Figure 4.2(a)-(c) presents the wire-frame results for Figure 4.1(a)-(c). In Figure 4.3 (a)-(c), the segmentation results to the original range image added by the effect of Gaussian noise (mean $=0$ and variance $=0.01$ ) are presented. Finally, Figure $4.4(\mathrm{a})$-(c) shows the obtained wire-frame representations to Figure 4.3(a)-(c). Table 1 shows a 
comparison of measure errors where $\varepsilon v$ is the 3D vertices' coordinates error, and $n v$ is the number of vertices. Surprisingly, the vertices' coordinates error $\varepsilon v$ for noisy sphere with $K=20$ is greater than the one for the sphere with $K=30$. This is due to the stability of the plane equations' computation.

\section{Conclusions}

This work presents a new integrated segmentation/representation process to range images. The main contributions of this work are: (1) A new approach for determining the optimal number of image regions; (2) A suitable representation that can be applied to both polyhedral and non-polyhedral objects. The advantages of the process described here are: (1) It is loosely dependent on threshold values; (2) It integrates image segmentation and image representation in a cooperative manner; (3) The final representation is robust; (4) It allows the computation of a concrete process quality measure; (5) It can be applied to polyhedral and non-polyhedral objects. There are three future directions to improve on the results of this work. The first one is to use the region reconstruction error to guide the clustering process to reduce the computational cost. The second research line is to compute a more significant representation quality measure based on the difference between the reconstructed surface and the original one. The last direction is to create a full 3-D object representation, by matching different views of the same object. These ideas are currently under study.

\section{References}

[1] P. Besl and R. Jain, 1988. "Segmentation through variable-order surface fitting". IEEE T-PAMI, Vol.10, No.2, pp.167-192.

[2] S. Bhandarkar and A. Siebert, 1992. "Integrating edge and surface information for range image segmentation". Pattern Recognition, Vol.25, No.9, pp.947-962.

[3] J. Chen, S. Castan, 1986. "An optimal linear operator for edge detection". Proc. of CVPR'86, Miami.

[4] R. Dubes and R.C. Jain, 1976. "Clustering techniques: the user's dilemma". Pattern Recognition Letters, Vol.8, pp.247-260.

[5] O.D. Faugeras and M. Herbert, 1987. "The representation, recognition, and positioning of 3D shapes from range data". Techniques for 3D Machine Perception, Ed. North Holland, Netherlands, pp.13-52.

[6] R.C. Gonzalez, R.E. Woods, 1992. Digital Image Processing. Addison-Wesley.

[7] J.F. Haddon, 1988. "Generalized threshold selection for edge detection", Pattern Recognition, Vol.3, pp.195-203.

[8] R. Hoffman and A. Jain, 1987. "Segmentation and classification of range images". IEEE T-PAMI, Vol.9, No.5, pp.608-620.

[9] R. Krishnapuram and A Munshi, 1991. "Cluster-based segmentation of range images using differential-geometric features". Optical Engineering, Vol.30, No.10, pp.1468-1478.

[10] B. Noble and J.W. Daniel, 1988. Applied Linear Algebra. Prentice-Hall Int.

[11] F.P. Preparata and M.I. Shamos, 1985. Computational Geometry: An Introduction. Springer-Verlag, NY-USA, 1985.

[12] N. Yokoya and M. Levine, 1989. "Range image segmentation based on differential geometry: a hybrid approach". IEEE T-PAMI, Vol.11, No.5, pp.643-649. 